\title{
Foreword to the invited impact paper on automatic software repair
}

\author{
Mark Harman
}

Published online: 12 July 2013

(C) Springer Science+Business Media New York 2013

The authors of this paper, Claire Le Goues, Stephanie Forrest and Westley Weimer, have been at the forefront of developments in automated software patching (also known as automated software repair). Their approach uses genetic programming, a search-based algorithm, in order to search for patches which fix software systems. The invited impact paper presented in this issue of the journal will serve as a useful introduction to the area for those new to automated software repair and a roadmap for future work for those actively engaged in the topic.

Impact papers are invited submissions to the journal, and they are reviewed speedily. An invited paper covers work that has already demonstrated an impact on the development of research, and so the review process starts from the presumption that the paper will ultimately be accepted. However, papers are reviewed by several expert reviewers (as normal), and authors are requested to respond to these reviewers' comments (as has happened in the case of this paper).

The authors' work on automated repair is well known and highly cited and has undoubtedly already had an impact on the direction of research work, both for work on search-based software engineering (SBSE) and also on work on automated software repair. It has received a distinguished paper award at the International Conference on Software Engineering (ICSE) and also the gold medal for human competitive results at the genetic and evolutionary computation conference (GECCO). It was therefore felt timely and appropriate that the authors be invited to submit an impact paper to the Journal.

This invited impact paper provides a survey of existing results in automated software repair, together with directions for future research in this area of SBSE. The results that have been achieved hitherto are, indeed, impressive, and we can look forward to a very bright future for this exciting and important area of research. I am confident that the reader will gain a great deal from this paper, whether or not they start out from a position of interest in either SBSE or automated software repair and so I commend the paper to the reader.

M. Harman $(\bowtie)$

Department of Computer Science, University College London, Gower Street, London WC1E 6BT, UK e-mail: mark.harman@ucl.ac.uk 